\title{
Clinical Benefit of Combination Treatment with 1,550 nm Fractional Laser and a New Wavelength at 1,927 nm on Photorejuvenation in Asian Patients
}

You Jin Lee

Jong Yoon Chung

Jae Hyung Lee

Jong Hee Lee

Joo Heung Lee

Department of Dermatology,

Samsung Medical Center

Sungkyunkwan University School of Medicine,

Seoul, Korea
Received June 17, 2014

Revised June 26, 2014

Accepted June 26, 2014

\section{Correspondence}

Jong Hee Lee

Department of Dermatology, Samsung Medical Center, Sungkyunkwan University School of Medicine, Seoul, Korea

Tel: $+82-2-3410-3549$

Fax: $+82-2-3410-3869$

E-mail: bell711dahanmail.net

(c) Korean Society for Laser Medicine and Surgery

(c) This is an open access article distributed under the terms of the Creative Commons Attribution NonCommercial License (http://creativecommons.org/ licenses/by-nc/3.0) which permits unrestricted noncommercial use, distribution, and reproduction in any medium, provided the original work is properly cited.

\section{Background and Objectives}

Fractional laser using a new wavelength of $1,927 \mathrm{~nm}$ is expected to work on superficial skin conditions, while a 1,550 nm fractional laser was proven to be effective in dermal remodeling. We administered the combination treatment using $1,550 \mathrm{~nm}$ with $1,927 \mathrm{~nm}$ in a single session to determine its clinical efficacy and safety in photorejuvenation.

\section{Materials and Methods}

Patients who received combination treatment were analyzed. Two blinded assessors graded the degree of improvement using photographs based on a quartile scale (0-3). Erythema and melanin index were measured on the same spot before and after treatment. Patients' satisfaction and adverse effects were also reported.

\section{Results}

A total of 25 patients were evaluated. Patients were followed up for up to 12 months ( $4.04 \pm 2.78$ months). The overall clinical improvement was 2.12 (Pigment improvement: $1.92 \pm 0.84$, wrinkle and skin texture; $1.66 \pm$ 0.54). Melanin index showed a substantial decrease after treatments. One patient experienced post inflammatory hyperpigmentation, which subsided with administration of a topical bleaching agent after six months.

\section{Conclusion}

Combination treatment with 1,550/1,927 nm may provide clinical benefit in Asian photoaged skin which contains a lot of pigmentation burden as well as wrinkles and other aging phenomenon.

\section{Key words}

Combination treatment; Photorejuvenation 


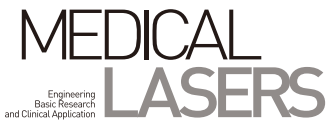

\section{INTRODUCTION}

Fractional photothermolysis is a new concept of treatment, in which precise arrays of microscopic treatment zones (MTZ) are produced in the dermis, stimulating a therapeutic response around them. Surrounding normal intact epidermis enables accelerated healing after treatments. ${ }^{1-3}$ Many studies have proven that this treatment modality can achieve desirable clinical results without serious adverse effects or longer downtime, which was the major limitation of ablative laser resurfacing. . $^{4-6}$

Nonablative fractional laser therapy at 1,550 nm was approved by FDA for the treatment of periorbital rhytides, skin resurfacing, acne, and surgical scars. ${ }^{5}$ It was also approved as an effective treatment modality for melasma and pigmented lesions. ${ }^{7.8}$ For the treatment of wrinkles, scars and other clinical conditions which require dermal remodeling, nonablative fractional laser treatment using 1,550 $\mathrm{nm}$ is a success without any doubt and lots of previous researches supported it. 2,5,9 However, there have been controversies on its clinical safety and efficacy for pigmentary conditions and melasma. Several studies ${ }^{2,7,8}$ insisted its clinical benefit on melasma or dyspigmentation in Caucasian skin, while clinical application on pigmentation in darker Asian skin has still limitation. $^{10-12}$

Recently, a novel 1,927-nm wavelength fractional laser has been developed and it was added to a 1,550nm erbium:glass (Er:Glass) fractional device. ${ }^{13,14}$ This new wavelength at 1,927 $\mathrm{nm}$ has a higher absorption coefficient for water than conventional 1,550 nm erbiumdoped fiber laser. This characteristics of wavelength implicates that the maximum depth of penetration at $1,927 \mathrm{~nm}$ would be about 200-300 $\mu \mathrm{m}$, while a 1,550 nm wavelength can reach down to 1,400-1,500 $\mu \mathrm{m}$ into the dermis. From this profile, it can be assumed that this new wavelength at 1,927 $\mathrm{nm}$ can target epidermal processes such as pigmentation and dyschromia, while conventional nonablative fractional laser at 1,550 nm works better on wrinkles and scars. ${ }^{15}$

Photoaged skin is a condition showing a combination of wrinkles, tactile roughness, telangiectasias and dyspigmentation. When we consider the treatment of photoaging in Asian skin, dyspigmentation and postinflammatory hyperpigmentation always matters. ${ }^{4}$ The modality to treat skin pigmentation, texture and wrinkles at the same time, is a must to ensure patient higher satisfaction. Therefore, dermal remodeling with $1,550 \mathrm{~nm}$ fractional laser treatment as well as improvement of superficial pigmentation and skin tones targeted by $1,927 \mathrm{~nm}$ thulium fiber fractional laser in a single session can be a promising treatment modality especially for photorejuvenation in Asian patients.

This study was performed to evaluate the clinical benefit and safety of combination treatment with 1,550 and 1,927 $\mathrm{nm}$ fractional photothermolysis in Asian photoaged skin.

\section{MATERIALS AND METHODS}

\section{Subjects}

Patients who received 1,550 and 1,927 nm combination treatment for their photoaged skin were included in the study. All patients were treated by one laser expert in a single institution.

Cases and photographs were reviewed retrospectively using medical records under the approval of Institutional Review Board. Patients who had been treated with any fillers or botox injection, and other photorejuvenation procedures within prior 6 months before enrollment were excluded in the analysis.

\section{Treatment procedures}

After 30 minutes of topical anesthesia, patients were treated first with 1,550 $\mathrm{nm}$ on the whole face and 1,927 $\mathrm{nm}$ fractional laser was performed on top of it using Fraxel Re:Store DUAL (Solta Medical, Hayward, CA). The treatment settings for $1,550 \mathrm{~nm}$ were $30 \mathrm{~mJ}$, treatment level 8 (total coverage 23\%), 8 passes and only 4 passes of it were delivered. Afterwards, 1,927 treatment was performed with a fluency of $10 \mathrm{~mJ}$, treatment level 3 (total coverage $30 \%$ ), and 4 passes. If patients wanted more treatment, the second treatment session was performed after two months, in which treatment settings were adjusted according to the residual clinical condition. For example, patients who had more residual pigmentation, the treatment parameter of 1,550 $\mathrm{nm}$ was set same as the first treatment session. For 1,927 nm treatment, fluence was adjusted according to skin thickness of patients (5-10 $\mathrm{mJ}$ ). The treatment level was set at 3 (total coverage 30\%), and passes were set at 8 passes. When $1,927 \mathrm{~nm}$ fractional laser was applied at the $2^{\text {nd }}$ treatment sessions, first 4 passes were done and after waiting for about 10 minutes with icepacks on the treated area, the rest 4 passes continued if mild scabs were not noticed or erythema was not intense enough. For patients with more wrinkles and skin roughness at the second treatment session, the same treatment parameter and settings as the first treatment session of 1,550 nm were applied. However, 8 passes were delivered on patients. 
For 1,927 $\mathrm{nm}$ treatment, the parameter was set at the fluence of $5 \mathrm{~mJ}$, treatment level 3 and 4 passes. A cooling device (Zimmer ElektromedizinCryo 5 device, Zimmer Medizin Systems, Irvine, CAl was used to alleviate pain and discomfort during treatment procedures. After treatment, an icepack with a cooling mask was applied on all patients for 20 minutes. Patients were instructed to avoid sun exposure and use topical moisturizer containing tranexamic acid two or three times a day until microcrust peeled off naturally. They were also educated to put on a broadspectrum sunscreen. Patients were asked to return according to the recommended follow-up regimen.

\section{Evaluation}

Photographs were taken at each visit. Two blinded assessor evaluated the degree of improvement using a quartile grading scale using photographs They scored overall improvement of photoaged skin as well as pigmentation, and textures and wrinkles in detail $10=$ worse or less than $25 \%$ improvement, $1=25-50 \%, 2=$ $51 \%-75 \%, 3=76-100 \%$ improvement). Erythema and melanin index were measured on the most prominent area of both zygomata, using skin color measuring device (Mexameter, MX18, Courage \& Khazaka, Electronic $\mathrm{GmbH}$, Clogne, Germanyl at every visit. Patients rated subjective satisfaction using a quartile scale $10=$ not satisfied, 1 = satisfied, 2 = very satisfied, 3 = extremely satisfied).

Adverse events were documented le.g. persistent erythema, edema, sever pain, crusting, infection, pigmentary changes, scarring).

\section{Statistical analysis}

Clinical data were compared using the Wilcoxon signed rank test. Paired $t$-test was used for the comparison of

Table 1. Degree of improvement using a quartile grading scale (0-3: $0=$ worse or less than $25 \%$ improvement, $1=25-50 \%, 2=51 \%$ $75 \%, 3=76-100 \%$ improvement). Patients who were treated twice were included in Group A. Group B means patients with only one treatment. " total" means all patients who received combination treatment. Patients also rated subjective satisfaction $(0=$ not satisfied, $1=$ satisfied, 2 = very satisfied, $3=$ extremely satisfied)

\begin{tabular}{lccc}
\hline & Group A $(n=12)$ & Group B $(n=13)$ & Total $(n=25)$ \\
\hline F/U (months) & $4.33 \pm 2.05$ & $3.77 \pm 3.29$ & $4.04 \pm 2.78$ \\
Overall improvement & $2.25 \pm 0.56^{*}$ & $2.00 \pm 0.91$ & $2.12 \pm 0.75$ \\
Pigment & $2.00 \pm 0.82$ & $1.85 \pm 0.90$ & $1.92 \pm 0.84$ \\
Textures/Wrinkles & $2.00 \pm 0.41^{*}$ & $1.35 \pm 0.47$ & $1.66 \pm 0.54$ \\
Patient's satisfaction & $2.33 \pm 0.62^{*}$ & $1.85 \pm 0.99$ & $2.08 \pm 0.84$ \\
\hline
\end{tabular}

*means $p<0.05$ Group A vs Group B, Wilcoxon signed rank test. erythema and melanin index. Statistical analysis was performed with SPSS software version 19.0 (SPSS Inc., Chicago, IL). All tests were assessed at an $\alpha=0.05$ significant level. $p<0.05$ is considered to be statistically significant. Values were documented as means \pm standard deviations.

\section{RESULTS}

A total of 25 healthy Korean patients (mean age: 51.5 years, standard deviation: 8.5 years, range: $43-72$ years, $M: F=4: 21$ l with Fitzpatrick skin types III-IV were included in the analysis. Twelve patients received two treatment sessions and the others underwent one treatment session. The follow up visit varied from 1 month to 12 months ( $4.04 \pm 2.78$ months).

Overall improvement in all patients was marked as 2.12 \pm 0.75 . Pigment improvement was evaluated as $1.92 \pm 0.84$ and wrinkles/textural smoothness as $1.66 \pm 0.54$. Patients who received two times of combination treatment appeared to show better clinical results. Patients' satisfaction tended to be higher in patients treated twice, too (Table 1, Fig. 1, 2, 3).

Erythema and melanin index were measured in ten patients who received two treatment sessions. These ten patients were followed up for $4.70 \pm 0.81$ months after the second treatment. Baseline erythema index was $201.3 \pm$ 37.2 and it dropped down to $180.2 \pm 41.8$ at 1 month after the first treatment. At last follow up, it was measured as $191.8 \pm$ 43.7. Considerable reduction in melanin index after the first treatment was noticed (from $192.9 \pm 28.3$ to $150.2 \pm 22.6$. . At last follow up after the second treatment,
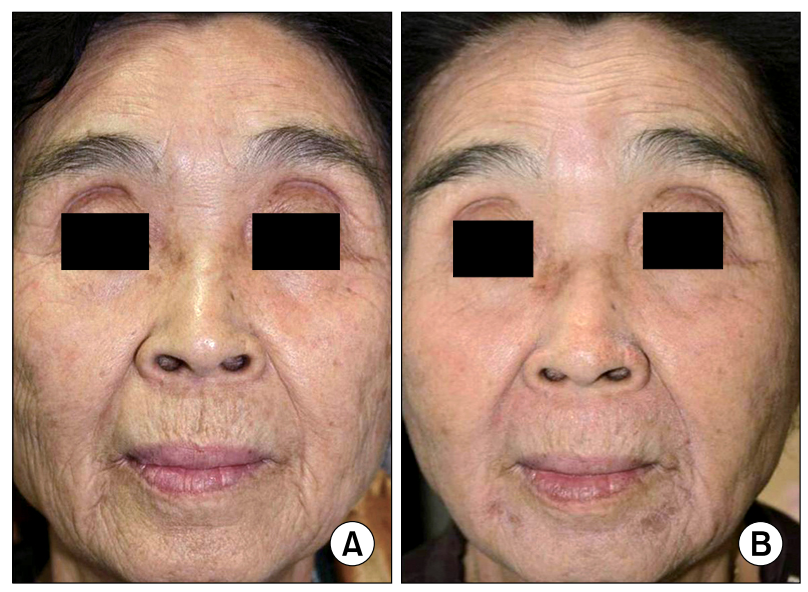

Fig. 1. She was treated twice with $1,550 / 1,927 \mathrm{~nm}$ combination treatment. Pigmentation as well as wrinkles and skin texture improved significantly after two treatment sessions. (A) Before treatment. (B) 2 months after the second treatment. 


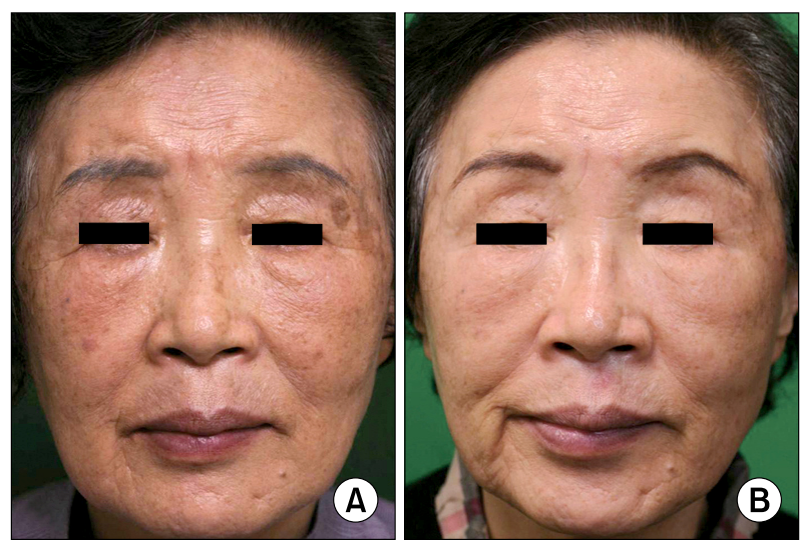

Fig. 2. A patient who received one treatment session of 1,550/1,927 combination. Considerable improvement in pigmentation was noticed after treatment. (A) Before treatment (B) 6 months after the treatment.

it showed substantial improvement of pigmentation compared with baseline and after first treatment (138.0 \pm 23.7) (Table 2).

Nineteen out of 25 patients reported that they were very or extremely satisfied with the treatment. One patient complained of transient pigment darkening after treatment and she did not want to get through another treatment session. She was advised to use topical $4 \%$ hydroquinone every night and her postinflammatory hyperpigmentation improved at 6 months follow up.

\section{DISCUSSION}

A lot of studies ${ }^{1-3,16,17}$ support fractional photothermolysis as an effective and safe treatment for photodamaged skin. Wanner et $\mathrm{al}^{5}$ reported that this nonablative 1,550 fractional laser worked on facial and non facial photodamage, rhytides and even dyspigmentation with low side effect profile. In the early era of fractional photothermolysis, it was expected that this fractional approach creates columns of thermal injury and melanin pigments are also successfully eliminated through these precise microscopic epidermal dermal necrotic debris (MEND) after treatment. ${ }^{6,16}$ Early studies focused on its clinical efficacy for dyspigmentation and even melasma. ${ }^{7,8,11}$ However, disappointing results were reported in pigmentary condition especially in darker skinned patients. ${ }^{12,18}$ Wind and colleagues ${ }^{10}$ even protested that topical therapy with triple combination cream remains the gold standard treatment of melasma compared with fractional photothermolysis in a randomized controlled split face study.

Fractional photothermolysis using a new wavelength at

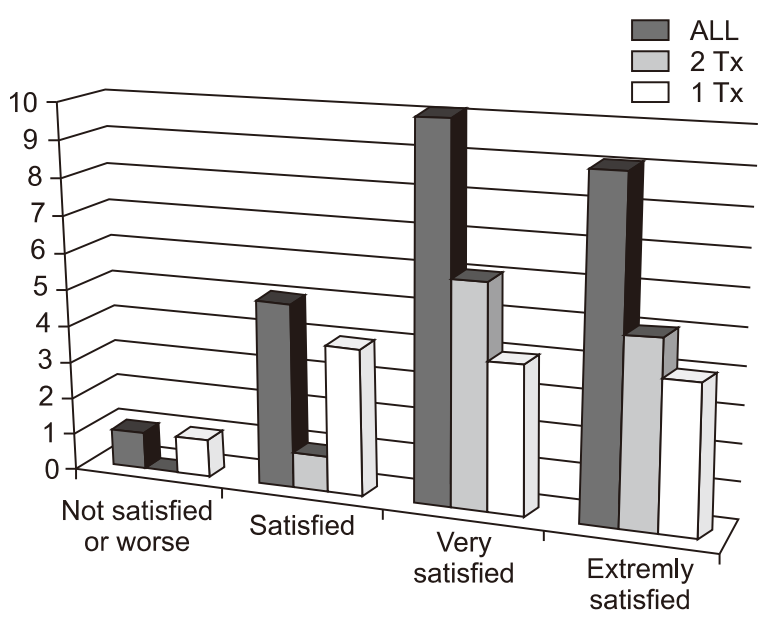

Fig. 3. Patients.

Table 2. Erythema and Melanin index were measured in ten patients who received two treatment sessions of $1,550 / 1,927 \mathrm{~nm}$. Decrease in melanin index after treatments was noticeable

\begin{tabular}{lcl}
\hline & Erythema Index & Melanin Index \\
\hline Before treatment & $201.3 \pm 37.2$ & $192.9 \pm 28.3$ \\
1 month after the first treatment & $180.2 \pm 41.8$ & $150.2 \pm 22.6^{*}$ \\
The last follow-up after & $191.8 \pm 43.7$ & $138.0 \pm 23.7^{*-}$ \\
$\quad$ the second treatment & & \\
\hline
\end{tabular}

$* p<0.01$ vs before treatment, paired t-test.

$\dagger p<0.05$ vs 1 month after the first treatment, paired $t$-test.

$1,927 \mathrm{~nm}$ has been studied in recent years. Higher water absorption at this wavelength confers a greater ability to target superficial conditions such as pigmentation and dyschromia. ${ }^{13,14}$ Several clinical studies showed that it can offer favorable results on macular seborrheic keratosis, and melasma. ${ }^{14,18,19}$ Lee and colleagues 18 demonstrated clinical improvement of photodamaged skin and melasma in Asian patients using 1,927 nm thulium fiber fractional laser. In their study, ${ }^{18}$ they reported that wrinkles, laxities and skin texture improved after treatment. However, the focus of clinical outcome was rather an improvement of pigmentation. ${ }^{18}$ Photoaged skin has many aspects including discrete pigmentary changes as well as rhytides, surface roughness and skin laxities which definitely need to dermal remodeling and deep tissue reaction. Therefore, we tried to figure out whether a combination using 1,550 and 1,927 nm fractional photothermolysis is safely applied in a single session for photorejuvenation in Asian patients. For setting the appropriate treatment parameter, a split face pilot study comparing 1,550 nm solo treatment with 1,550/1,927 combination treatment was performed ahead of this study. ${ }^{20}$ 
This study showed this combination modality yielded low adverse effect profile on patients for photorejuvenation. We did not use higher coverage like $60-70 \%$ in previous studies because of possible risk of postinflammatory hyperpigmentation. ${ }^{19,21}$ Under the parameter and procedure applied in this study, the combination of $1,550 / 1,927 \mathrm{~nm}$ fractional photothermolysis appeared to provide patients with expected synergistic effects. Only one patient who got through pigment darkening after treatment was not satisfied. Others were satisfied with treatment outcomes. The higher score of satisfaction was observed in patients who received two treatment sessions. Overall improvement as well as textures and wrinkles obtained higher score in them. Interestingly, improvement in pigmentation failed to get statistically significant higher score in patients who were treated twice. As shown in previous studies ${ }^{13-15,18}$ using a 1,927 nm fractional laser, superficial condition like pigmentation responded excellent after treatment while deep dermal remodeling which reflects improvement of skin textures and wrinkles may take time and require more treatment sessions.

In reviews of previous studies, Kearney et al ${ }^{22}$ reported clinical benefit of the combination treatment with 1,550 Erbium glass fractional laser and intense pulsed light in single session over each single treatment modality. Based on the knowledge from this study, ${ }^{22}$ we authors performed the same treatment using exactly same devices and parameters on patients with photodamaged skin. When we applied intense pulsed light, the fluence was lowered by 10 to $15 \%$ compared with their study because of higher risk of postinflammatory hyperpigmentation in Asian skin. ${ }^{16}$ The results were not satisfactory at all. Three out of 4 patients returned with postinflammatory hyperpigmentation (data was not shown here). Kearney et $\mathrm{al}^{22}$ applied a treatment parameter of 1,550 nm fractional laser as $30 \mathrm{~mJ}$ - level 8 (23\% coverage) -8 passes. Considerable erythema usually achieved after 1,550 nm fractional laser treatment with this parameter. Intense pulsed light application right after this treatment might increase the risk of postinflammatory hyperpigmentation in darker skinned patients. However, 1,550/1,927 combination treatment in this study showed rather safe results in Asian photoaged skin. Fractional photothermolysis using a wavelength at 1,927 nm did not respond to skin erythema unlike intense pulsed light. Therefore, it is assumed that patients with darker skin type can tolerate this combination treatment better.

A combination of different lasers and light sources for concomitant treatments gives way to a potential area of advancement in the clinical field. Many dermatologists are performing combined treatments in their daily practices to enhance clinical outcomes, but little has been published until now. This study has its own value from this point of view. Although this was not prospective study or did not have any control like 1,927 or 1,550 single treatment, the result turned out very promising.

In conclusion, the combination using 1,550/1,927 nm fractional laser in a single session may provide another safe and effect treatment modality for photorejuvenation in Asian patients. Prospective studies to validate its use or a comparative study between combination and solo treatment would be needed.

\section{REFERENCES}

1. Laubach HJ, Tannous Z, Anderson RR, Manstein D. Skin responses to fractional photothermolysis. Lasers Surg Med 2006:38:142-9.

2. Taub AF. Fractionated delivery systems for difficult to treat clinical applications: acne scarring, melasma, atrophic scarring, striae distensae, and deep rhytides. J Drugs Dermatol 2007;6:1120-8.

3. Tierney EP, Kouba DJ, Hanke CW. Review of fractional photothermolysis: treatment indications and efficacy. Dermatol Surg 2009;35:1445-61.

4. Lee SM, Kim MS, Kim YJ, Won CH, Lee MW, Choi JH, et al. Adverse events of non-ablative fractional laser photothermolysis: a retrospective study of 856 treatments in 362 patients. J Dermatolog Treat 2014;25:304-7.

5. Wanner M, Tanzi EL, Alster TS. Fractional photothermolysis: treatment of facial and nonfacial cutaneous photodamage with a 1,550-nm erbium-doped fiber laser. Dermatol Surg 2007;33: 23-8.

6. Manstein D, Herron GS, Sink RK, Tanner H, Anderson RR. Fractional photothermolysis: a new concept for cutaneous remodeling using microscopic patterns of thermal injury. Lasers Surg Med 2004;34:426-38.

7. Naito SK. Fractional photothermolysis treatment for resistant melasma in Chinese females. J Cosmet Laser Ther 2007;9:1613.

8. Rokhsar CK, Fitzpatrick RE. The treatment of melasma with fractional photothermolysis: a pilot study. Dermatol Surg 2005; 31:1645-50.

9. Ong MW, Bashir SJ. Fractional laser resurfacing for acne scars: a review. Br J Dermatol 2012;166:1160-9.

10. Wind BS, Kroon MW, Meesters AA, Beek JF, van der Veen JP, Nieuweboer-Krobotová L, et al. Non-ablative 1,550 nm fractional laser therapy versus triple topical therapy for the 
treatment of melasma: a randomized controlled split-face study. Lasers Surg Med 2010;42:607-12.

11. Katz TM, Glaich AS, Goldberg LH, Firoz BF, Dai T, Friedman PM. Treatment of melasma using fractional photothermolysis: a report of eight cases with long-term follow-up. Dermatol Surg 2010;36:1273-80.

12. Karsai S, Fischer T, Pohl L, Schmitt L, Buhck H, Jünger $M$, et al. Is non-ablative 1550-nm fractional photothermolysis an effective modality to treat melasma? Results from a prospective controlled single-blinded trial in 51 patients. J Eur Acad Dermatol Venereol 2012;26:470-6.

13. Polder KD, Mithani A, Harrison A, Bruce S. Treatment of macular seborrheic keratoses using a novel 1927-nm fractional thulium fiber laser. Dermatol Surg 2012;38:1025-31.

14. Polder KD, Bruce S. Treatment of melasma using a novel 1,927-nm fractional thulium fiber laser: a pilot study. Dermatol Surg 2012;38:199-206.

15. Weiss ET, Brauer JA, Anolik R, Reddy KK, Karen JK, Hale EK, et al. 1927-nm fractional resurfacing of facial actinic keratoses: a promising new therapeutic option. J Am Acad Dermatol 2013; 68:98-102.

16. Rahman Z, Alam M, Dover JS. Fractional Laser treatment for pigmentation and texture improvement. Skin Therapy Lett
2006;11:7-11.

17. Graber EM, Tanzi EL, Alster TS. Side effects and complications of fractional laser photothermolysis: experience with 961 treatments. Dermatol Surg 2008;34:301-5; discussion 5-7.

18. Lee HM, Haw S, Kim JK, Chang SE, Lee MW. Split-face study using a 1,927-nm thulium fiber fractional laser to treat photoaging and melasma in Asian skin. Dermatol Surg 2013; 39:879-88.

19. Ho SG, Yeung CK, Chan NP, Shek SY, Chan HH. A retrospective study of the management of Chinese melasma patients using a $1927 \mathrm{~nm}$ fractional thulium fiber laser. J Cosmet Laser Ther 2013;15:200-6.

20. Lee JH. New wavelength at 1,927 for Asian Skin. Lectures at: IMCAS Asia 2013; 2013 July 27-29; Singapore.

21. Niwa Massaki AB, Eimpunth S, Fabi SG, Guiha I, Groff W, Fitzpatrick R. Treatment of melasma with the 1,927-nm fractional thulium fiber laser: a retrospective analysis of 20 cases with long-term follow-up. Lasers Surg Med 2013;45:95101.

22. Kearney C, Brew D. Single-session combination treatment with intense pulsed light and nonablative fractional photothermolysis: a split-face study. Dermatol Surg 2012;38:1002-9. 